\title{
COMPONENTES DO BALANÇO DE ÁGUA NO SOLO COM CANA-DE-AÇÚCAR, COM E SEM ADUBAÇÃO NITROGENADA ${ }^{(1)}$
}

\author{
Alexsandro dos Santos Brito ${ }^{(2)}$, Paulo Leonel Libardi ${ }^{(3)} \&$ Pablo \\ Javier Ghiberto ${ }^{(2)}$
}

\begin{abstract}
RESUMO
O estudo do balanço de água no solo com cana-de-açúcar, cultivada em larga escala no Brasil, é essencial para o manejo agrícola, visando ao aumento de produtividade e à redução de riscos de poluição. Este estudo objetivou avaliar os componentes da equação do balanço de água em dois Latossolos cultivados com cana-de-açúcar (cana-planta), localizados nos Municípios de Jaboticabal e Pirassununga (São Paulo). Na área experimental de Jaboticabal, o estudo foi realizado entre os dias 30 de setembro de 2005 e 12 de julho de 2006 , e constou de dois tratamentos: $T_{1}$ (sem adubação nitrogenada) e $T_{2}\left(120 \mathrm{~kg} \mathrm{ha}^{-1} \mathrm{de} N\right)$. Em Pirassununga, o estudo foi feito entre os dias 28 de setembro de 2005 e 08 de junho de 2006, mas apenas o tratamento $\mathrm{T}_{2}$. O delineamento experimental foi o de blocos aleatórios, com quatro repetições. $O$ volume de controle utilizado para o balanço de água teve como limite superior a superfície do solo e como limite inferior outra paralela à primeira, localizada em profundidade de $0,90 \mathrm{~m}$. As variações de armazenagem, as entradas (precipitação pluvial e ascensão capilar) e as saídas de água (evapotranspiração real e drenagem interna) nesse volume de solo, nas áreas experimentais de Jaboticabal e Pirassununga, foram determinadas em 13 e 10 períodos, respectivamente. Ambas as áreas estudadas eram planas e não irrigadas. Na área de Jaboticabal, em todo o período, tanto a perda de água por drenagem interna quanto o ganho por ascensão capilar foram maiores no tratamento $\mathrm{T}_{2}(-$ $191,69 \mathrm{~mm} / 5,33 \mathrm{~mm}$ e $-238,37 \mathrm{~mm} / 24,60 \mathrm{~mm}$ para $T_{1}$ e $T_{2}$, respectivamente); não houve diferença significativa de produtividade $\left(145\right.$ e $146 \mathrm{t} \mathrm{ha}^{-1}$ para $\mathrm{T}_{1}$ e $\mathrm{T}_{2}$, respectivamente), nem de evapotranspiração real $\left(1.058 \mathrm{~mm}\right.$ para $T_{1}$ e $1.028 \mathrm{~mm}$ para o $T_{2}$ ) entre os tratamentos nesse ciclo de cana-planta, fazendo com que as plantas não adubadas apresentassem a mesma eficiência do uso da água (14 $\left.\mathrm{kg} \mathrm{m}^{-3}\right)$ que as adubadas. Na área de Pirassununga, a drenagem interna e a ascensão capilar foram de $-110,9$ e $0,67 \mathrm{~mm}$, respectivamente, e a eficiência de uso da água
\end{abstract}

\footnotetext{
(1) Parte da Dissertação de Mestrado do primeiro autor. Projeto financiado pela FAPESP (2002/10.534-8). Recebido para publicação em setembro de 2008 e aprovado em fevereiro de 2009.

(2) Programa de Pós-graduação em Solos e Nutrição de Plantas, Escola Superior de Agricultura "Luiz de Queiroz" - ESALQ. Universidade de São Paulo - USP. Av. Pádua Dias 11, CEP 13418-900 Piracicaba (SP). Bolsista CNPq. E-mail: alexsb@esalq.usp.br; pjghiber@esalq.usp.br

(3) Departamento de Ciências Exatas, ESALQ. E-mail: pllibard@esalq.usp.br
} 
$13 \mathrm{~kg} \mathrm{~m}^{-3}$. Portanto, pode-se concluir que (a) nos períodos de alta pluviosidade, a drenagem interna foi significativa, alertando para possíveis problemas no manejo em relação a uma potencial de contaminação do lençol freático, e (b) tanto a evapotranspiração real quanto à eficiência do uso da água foram da mesma ordem de grandeza, independentemente da adubação nitrogenada, e a armazenagem de água no solo foi praticamente a mesma no início e no fim do ciclo estudado.

Termos de indexação: Drenagem interna, ascensão capilar, evapotranspiração real, tensiômetro.

\title{
SUMMARY: WATER BALANCE COMPONENTS IN SOILS CROPPED TO SUGARCANE, WITH AND WITHOUT NITROGENFERTILIZATION
}

\begin{abstract}
The water balance study in soils with sugarcane, cropped in large areas in Brazil, is essential for the agricultural management to increase the crop productivity and to reduce pollution risks. The objective of this research was to evaluate soil water balance equation components of two Oxisols under sugar-cane (first crop), in the counties of Jaboticabal and Pirassununga, State of São Paulo, Brazil. The study was carried out in an experimental area in Pirassununga $\left(21^{\circ} 55^{\prime} 54^{\prime} \mathrm{S}, 47^{\circ} 10^{\prime} 54^{\prime \prime} \mathrm{W}, 650 \mathrm{~m}\right)$, for only one level (120 $\left.\mathrm{kg} \mathrm{ha} \mathrm{h}^{-1}\right)$ of nitrogen, in four replications. In Jaboticabal (21 ${ }^{\circ} 19^{\prime} 98^{\prime}$ ' $\mathrm{S}, 48^{\circ} 19^{\prime} 03^{\prime} \mathrm{W}, 600 \mathrm{~m}$ ), two

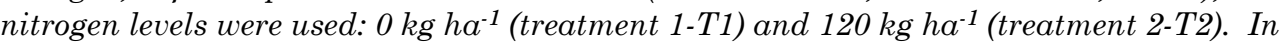
Pirassununga, the experiments were evaluated between September 28, 2005 and June 08, 2006 and, in Jaboticabal, between September 30, 2005 and July 12, 2006. The upper limit of the control volume used for the water balance was the soil surface and the lower limit a surface at a soil depth of $0.90 \mathrm{~m}$, parallel to the soil surface. Soil water storage variations, soil water inputs (rain and capillary rise) and soil water outputs (internal drainage and actual evapotranspiration) were determined in 13 and 10 monitoring periods, in Jaboticabal and Pirassununga, respectively. Both studied areas were plane and non irrigated. In the area in Jaboticabal, both internal drainage and capillary rise were greater in treatment $T_{2}$ ($191.69 \mathrm{~mm} / 5.33 \mathrm{~mm}$ and $-238.37 \mathrm{~mm} / 24.60 \mathrm{~mm}$ for $T_{1}$ and $T_{2}$, respectively) for the entire period; there was no significant difference in yield (145 and $146 \mathrm{t} \mathrm{ha} \mathrm{h}^{-1}$ for $T_{1}$ and $T_{2}$, respectively) and also in actual evapotranspiration (1058 $\mathrm{mm}$ for $T_{1}$ and $1028 \mathrm{~mm}$ for $T_{2}$ ) between the treatments in this sugar-cane cycle, so that the water use efficiency of plants without nitrogen fertilization was the same $\left(14 \mathrm{~kg} \mathrm{~m}^{-3}\right)$ as of those with nitrogen fertilization. In Pirassununga, internal drainage and capillary rise were -110.9 and $0.67 \mathrm{~mm}$, respectively and the water use efficiency $13 \mathrm{~kg} \mathrm{~m}^{-3}$. It can therefore be concluded that (a) internal drainage was relevant in the high rainfall periods, which warns for possible problems in soil management due to the risk of groundwater contamination, and (b) the actual evapotranspiration and the water use efficiency were in the same order of magnitude, independently of nitrogen fertilization; and soil water storage was practically the same from the beginning to the end of the studied cycle.
\end{abstract}

Index terms: internal drainage, capillary rise, actual evapotranspiration, tensiometers.

\section{INTRODUÇÃO}

Atualmente, o Brasil é o maior produtor mundial de cana-de-açúcar (Saccharum spp.), com uma área cultivada de 5.571.400 ha e uma produção de 410.983.008 t. O Estado de São Paulo se destaca como o maior produtor nacional, participando com $60,34 \%$ da safra 2005/2006 (FNP, 2006).

O aumento de 725.000 ha cultivados com cana-deaçúcar, observado entre 2000 e 2004, é um indicativo da necessidade de uma matriz energética alternativa e que englobe o conceito de sustentabilidade em sua concepção, etapas e processos (FNP, 2006).
Esse crescimento no setor sucroalcooleiro gera, porém, questionamentos sobre os impactos no ambiente, a necessidade do aumento da produtividade e maior eficiência no uso da água pela cultura. Nesse aspecto, o método que possibilita o estudo mais detalhado das condições hídricas em que a cultura se desenvolveu é o que quantifica os processos da equação do balanço da água no solo (Brunini et al., 1981; Libardi \& Saad, 1994; Antonino et al., 2000; Cintra et al., 2000; Timm et al., 2002; Cruz et al., 2005a). Esta equação mostra que a soma algébrica, durante dado período, de todas as entradas (consideradas positivas) e saídas (negativas) de água em um volume de controle de solo, submetido às peculiaridades dos fenômenos 
meteorológicos, dos atributos do solo e das características genéticas da espécie ou mesmo da variedade vegetal, resulta no saldo ou balanço de água no solo no período. Considera-se esse volume, no campo, delimitado por duas superfícies paralelas: a interface solo-atmosfera (limite superior do volume ou simplesmente superfície do solo) e uma superfície horizontal localizada à profundidade do sistema radicular do cultivo (limite inferior do volume).

Alguns estudos de adubação nitrogenada em canade-açúcar demonstram que essa cultura responde com maior produção de colmos e rendimento de açúcar e que essa adubação associada à incorporação de resíduos culturais ao solo faz com que a planta utilize maior quantidade total de N (Trivelin et al., 2002), principalmente a partir da segunda soca, quando a fertilização propicia, possivelmente, a recomposição do vigor da soqueira (Vitti et al., 2007). A interação entre nutrientes, como é o caso de $\mathrm{N}$ e $\mathrm{K}$, também influencia significativamente o rendimento de colmos, açúcar e álcool (Moura et al., 2005), evidenciando a importância do balanço nutricional na eficiência de produção de fitomassa.

A influência do consumo de água e da adubação nitrogenada no crescimento e no rendimento tem sido relatada para muitas culturas (Araújo et al., 1999; Wiedenfeld, 2000; Santos et al., 2002). Entretanto, com relação à cana-de-açúcar, são poucos os estudos que quantificam os processos envolvidos na equação do balanço de água no solo (ou componentes do balanço de água no solo) com a adubação nitrogenada. Além disso, há a preocupação quanto à contaminação dos mananciais com nutrientes, principalmente o $\mathrm{NO}_{3}$ (Stewart et. al., 2006). Nesse caso, uma das maneiras de quantificar esse potencial poluente é por meio da drenagem interna.

Como se trata de uma cultura que se tornou a principal na produção de energia limpa e que vem aumentando a área cultivada, é importante a realização de pesquisas quanto ao regime hídrico, de forma a identificar soluções de manejo que possibilitem a melhoria da produtividade. Portanto, o objetivo do trabalho foi avaliar os processos envolvidos na equação do balanço de água em dois solos cultivados com cana-de-açúcar, um com e o outro com e sem adubação nitrogenada. Os processos avaliados foram variação de armazenagem, precipitação pluvial, drenagem interna, ascensão capilar e evapotranspiração real.

\section{MATERIAL E MÉTODOS}

O estudo foi realizado na Usina Santa Adélia, localizada no município de Jaboticabal, e na Usina São Luiz, localizada no município de Pirassununga, grandes regiões produtoras de cana-de-açúcar. As coordenadas geográficas da área experimental da Usina Santa Adélia são $21^{\circ} 19^{\prime} 98$ " S, $48^{\circ} 19^{\prime} 03^{\prime \prime W}$ e $600 \mathrm{~m}$ e as da São Luiz, $21^{\circ} 55^{\prime} 54^{\prime}$ S, $47^{\circ} 10^{\prime} 54$ " W e $650 \mathrm{~m}$. Os climas dos dois locais são do tipo Aw e Cwa, respectivamente, segundo a classificação de Köppen. O valor médio de precipitação pluvial anual para o município de Jaboticabal, calculado de uma série histórica (1956 a 1995) é de $1.428 \mathrm{~mm}$, e para o período deste estudo a média foi de $1.345 \mathrm{~mm}$, sendo o período chuvoso compreendido entre outubro e março, com médias de 140, 151, 248, 240, 211 e $168 \mathrm{~mm}$, respectivamente (Centurion \& Andrioli, 2000). Já para Pirassununga, a média anual (série histórica de 1956-1972) é de $1.373 \mathrm{~mm}$, e para o período deste estudo foi de $1.118 \mathrm{~mm}$, possuindo o mesmo período chuvoso de Jaboticabal e com médias de 136, 124, 250, 253, 218 e $127 \mathrm{~mm}$ para os meses de outubro a março, respectivamente (SIGRH, 2008).

A instalação do experimento, na Usina Santa Adélia, foi realizada entre os dias 4 e 8 de abril de 2005 e na Usina São Luiz entre os dias 21 e 24 de fevereiro de 2005, quando se procedeu à sulcagem (espaçamento de 1,5 m entrelinhas), à marcação das parcelas e dos carreadores, à adubação e ao plantio da cana-de-açúcar. A variedade de cana-de-açúcar utilizada foi a SP81-3250 e a adubação foi realizada manualmente, aplicando no fundo do sulco o cloreto de potássio, o superfosfato simples e a uréia como fontes de $\mathrm{K}_{2} \mathrm{O}\left(120 \mathrm{~kg} \mathrm{ha}^{-1}\right), \mathrm{P}_{2} \mathrm{O}_{5}\left(120 \mathrm{~kg} \mathrm{ha}^{-1}\right)$ e de $\mathrm{N}$ (variando a dose conforme o tratamento), respectivamente.

O delineamento experimental foi em blocos aleatorizados, com dois tratamentos $\left(\mathrm{T}_{1}-\right.$ sem adubação nitrogenada e $\mathrm{T}_{2}-120 \mathrm{~kg} \mathrm{ha}^{-1} \mathrm{de} \mathrm{N}$ ) e quatro repetições. Entretanto, na área experimental de Pirassununga, o estudo foi realizado apenas no tratamento $\mathrm{T}_{2}$. As áreas experimentais tinham $31.500 \mathrm{~m}^{2}$, e as parcelas possuíam $1.080 \mathrm{~m}^{2}$, constituídas por 28 linhas de plantas, com comprimento de $15 \mathrm{~m}$.

Para a medida da produtividade foram selecionadas quatro parcelas por bloco para cada tratamento, sendo colhidas quatro linhas de plantas com $15 \mathrm{~m}$ de comprimento.

Os solos de Jaboticabal e Pirassununga foram classificados como Latossolo Vermelho distrófico típico (Embrapa, 1999), Arenic Kandiustults (Soil Survey Staff, 2003), e Latossolo Vermelho-Amarelo distrófico, Typic Haplustox, respectivamente. A caracterização física dos solos (Quadro 1) foi feita a partir de amostras deformadas (análise granulométrica e densidade das partículas) e indeformadas (densidade do solo, obtida por meio de um amostrador tipo Uhland: cilindro de $0,05 \mathrm{~m}$ de altura e $0,05 \mathrm{~m}$ de diâmetro) retiradas de uma trincheira aberta na área de bordadura dos experimentos. A porosidade total foi calculada a partir das densidades do solo e das partículas.

A análise granulométrica foi feita pelo método da pipeta (Gee \& Or, 2002) e a densidade das partículas, pelo método do deslocamento de gás (Flint \& Flint, 2002), utilizando um picnômetro a gás, modelo ACCUPYC 1330 (Micromeritics Instrument Corporation ${ }^{\circledR}$ ). 
Quadro 1. Caracterização física do perfil dos solos da área experimental de Jaboticabal e de Pirassununga

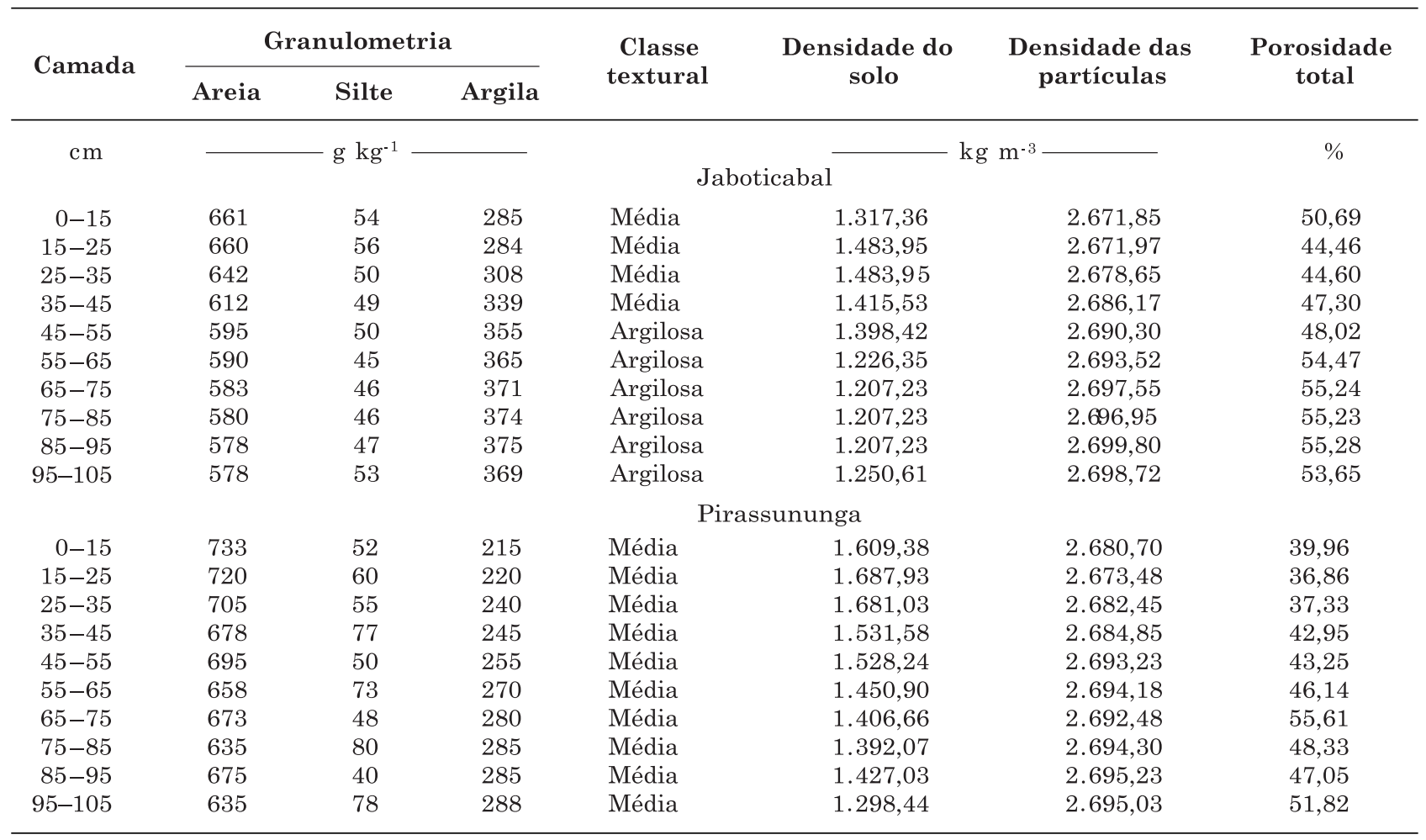

A caracterização hídrica dos solos consistiu na determinação das funções $\theta\left(\phi_{m}\right)$ e $K(\theta)$, em que $\phi_{m}=$ potencial mátrico, $K=$ condutividade hidráulica e $\theta=$ conteúdo de água volumétrica do solo. A função $\theta\left(\phi_{m}\right)$, para cada horizonte dos solos, foi determinada por meio de funis de Haines, para as tensões de 0,5; 1,$0 ; 3,0 ; 5,0 ; 7,0 ;$ e 10,0 kPa, e de câmaras de pressão de Richards, para as tensões de 30,0; 50,0; 70,0; e $100,0 \mathrm{kPa}$. Na área de Jaboticabal, foi determinado o conteúdo de água retida sob a tensão de $1.500 \mathrm{kPa}$, considerado como ponto de murchamento permanente (PMP). Os dados obtidos foram utilizados para determinar os parâmetros da equação (1):

$$
\theta\left(\phi_{m}\right)=\theta_{r}+\frac{\theta_{s}-\theta_{r}}{\left[1+\left(\alpha \times \phi_{m}\right)^{n}\right]^{m}}
$$

proposta por van Genuchten (1980), na qual $\theta_{r}$ é a conteúdo de água residual, $\theta_{s}$ é a conteúdo de água de saturação e $\alpha, \mathrm{m}$ e $n$ são parâmetros de ajuste. No quadro 2 , encontram-se os valores dos parâmetros da equação (1), bem como os coeficientes de determinação $\left(\mathrm{r}^{2}\right)$ para ambos os solos estudados. Na determinação dessa função, foram utilizadas amostras indeformadas retiradas da mesma trincheira e com o mesmo amostrador utilizado na determinação da densidade do solo.

A função $K(\theta)$, para a profundidade de $0,9 \mathrm{~m}$, foi determinada no campo, pelo método do perfil instantâneo (Watson, 1966; Hillel et al., 1972; Libardi et al., 1980), também na área de bordadura dos experimentos. Os dados obtidos foram ajustados à equação:

$$
K(\theta)=K_{0} \exp g r\left(\theta-\theta_{0}\right)
$$

na qual $K_{0}$ é a condutividade hidráulica do solo no tempo zero de redistribuição, conforme o método do perfil instantâneo, $\gamma$ é um parâmetro de ajuste e $\theta_{0}$ é a conteúdo de água volumétrica do solo no tempo zero de redistribuição. Para a área de Jaboticabal: $K_{0}=$ $792,86 \mathrm{~mm} \mathrm{dia}^{-1} ; \gamma=46,77$ e $\theta_{0}=0,366 \mathrm{~m}^{3} \mathrm{~m}^{-3} \mathrm{e} \mathrm{r}^{2}=$ 0,99. Para a área de Pirassununga: $K_{0}=$ $443,30 \mathrm{~mm} \mathrm{dia}^{-1} ; \gamma=52,23$ e $\theta_{0}=0,365 \mathrm{~m}^{3} \mathrm{~m}^{-3} \mathrm{e} \mathrm{r}^{2}=$ 0,98 .

\section{A equação do balanço de água no solo}

Em Jaboticabal, a quantificação dos processos que constituem a equação do balanço de água no solo foi realizada em 13 períodos compreendidos entre 30 de setembro de 2005 e 12 de julho de 2006, e em Pirassununga entre 28 de setembro de 2005 e 08 de junho de 2006, perfazendo 10 períodos (Quadro 3).

A equação do balanço de água no solo utilizada neste estudo foi:

$$
P+1+D+A C+E T r+R=\Delta h_{z}
$$


na qual: P - precipitação pluvial; I - irrigação; D drenagem interna; AC - ascensão capilar; ETr evapotranspiração real; $\mathrm{R}$ - deflúvio superficial e $\Delta \mathrm{h}_{\mathrm{z}}$ - variação da armazenagem de água, todos medidos ou calculados em mm. As áreas experimentais foram manejadas em sistema de sequeiro e apresentavam declividades inferiores a $5 \%$, pelo que os componentes I e $\mathrm{R}$ foram considerados iguais a zero na equação (3).

A precipitação pluvial foi medida por um pluviômetro, modelo paulista, instalado ao lado dos experimentos.

Para a determinação da drenagem interna (D) e ascensão capilar (AC) diárias foram instalados, em todas as repetições, tensiômetros com vacuômetro tipo Bourdon, nas profundidades de 0,80; 0,90; e 1,0 m. Com as leituras dos tensiômetros e da curva de retenção a $0,90 \mathrm{~m}$, determinaram-se a condutividade hidráulica do solo nesta profundidade pela equação (2) e, com as dos outros dois tensiômetros, o gradiente de potencial total da água no solo na mesma profundidade, conforme a equação:

$$
\operatorname{Grad} \phi_{t}=\frac{\phi_{t 1}-\phi_{t 2}}{\Delta z}
$$

sendo $\phi_{t 1}$ e $\phi_{t 2}$ os potenciais totais da água nas profundidades de 0,8 e $1,0 \mathrm{~m}$, respectivamente, e $\Delta z$ a distância entre o centro das cápsulas porosas desses tensiômetros $(=0,20 \mathrm{~m})$.

A instalação dos tensiômetros foi realizada após o nivelamento do solo nas entrelinhas de plantio ("quebra-lombo"), daí as medidas terem sido iniciadas em setembro de 2005.

Conhecendo a condutividade hidráulica do solo e o gradiente de potencial total da água no solo, foi possível

Quadro 2. Parâmetros de ajuste da curva de retenção das áreas experimentais de Jaboticabal e Pirassununga

\begin{tabular}{|c|c|c|c|c|c|c|}
\hline \multirow{2}{*}{ Horizonte } & \multicolumn{5}{|c|}{ Parâmetro de ajuste da curva de retenção da água no solo } & \multirow{2}{*}{$\mathbf{r}^{2}$} \\
\hline & $\theta_{\mathrm{s}}$ & $\theta_{\mathrm{r}}$ & $\alpha$ & $\mathbf{m}$ & $\mathbf{N}$ & \\
\hline & \multicolumn{2}{|c|}{$\mathrm{m}^{3} \mathrm{~m}^{-3}$} & \multirow{2}{*}{\multicolumn{3}{|c|}{$\mathrm{kPa}^{-1}$}} & \\
\hline & & & & & & \\
\hline$A_{p}$ & 0,4740 & 0,1582 & 0,6735 & 0,2725 & 3,1798 & 0,99 \\
\hline $\mathrm{A}_{2}$ & 0,4262 & 0,1712 & 0,6259 & 0,2352 & 3,2171 & 0,99 \\
\hline $\mathrm{BA}$ & 0,4161 & 0,1888 & 0,5515 & 0,0835 & 7,7256 & 0,99 \\
\hline $\mathrm{B}_{\mathrm{w} 1}$ & 0,4297 & 0,1676 & 0,5227 & 0,1200 & 7,2554 & 0,99 \\
\hline \multirow[t]{2}{*}{$\mathrm{B}_{\mathrm{w} 2}$} & 0,4532 & 0,1594 & 0,4720 & 0,1807 & 4,4588 & 0,99 \\
\hline & \multicolumn{6}{|c|}{ Pirassununga } \\
\hline $\mathrm{A}$ & 0,372 & 0,183 & 0,133 & 2,269 & 0,749 & 0,99 \\
\hline $\mathrm{AB}$ & 0,344 & 0,088 & 0,534 & 3,192 & 0,007 & 0,99 \\
\hline $\mathrm{B}_{\mathrm{w} 1}$ & 0,389 & 0,134 & 0,490 & 8,915 & 0,070 & 0,99 \\
\hline $\mathrm{B}_{\mathrm{w} 2}$ & 0,405 & 0,130 & 0,349 & 1,775 & 0,454 & 0,99 \\
\hline
\end{tabular}

Quadro 3. Períodos de avaliação dos processos da equação do balanço de água no solo

\begin{tabular}{|c|c|c|c|c|c|c|c|}
\hline \multicolumn{4}{|c|}{ Jaboticabal } & \multicolumn{4}{|c|}{ Pirassununga } \\
\hline Período & Data inicial & Data final & Total de dias & Período & Data inicial & Data final & Total de dias \\
\hline 1 & $30 / 09 / 05$ & $18 / 10 / 05$ & 19 & 1 & $28 / 09 / 05$ & $17 / 10 / 05$ & 19 \\
\hline 2 & $18 / 10 / 05$ & $08 / 11 / 05$ & 21 & 2 & $17 / 10 / 05$ & $07 / 11 / 05$ & 21 \\
\hline 3 & $08 / 11 / 05$ & $05 / 12 / 05$ & 27 & 3 & $07 / 11 / 05$ & $05 / 12 / 05$ & 28 \\
\hline 4 & $05 / 12 / 05$ & $19 / 12 / 05$ & 14 & 4 & $05 / 12 / 05$ & $22 / 12 / 05$ & 17 \\
\hline 5 & $19 / 12 / 05$ & $13 / 01 / 06$ & 25 & 5 & $22 / 12 / 05$ & $10 / 01 / 06$ & 19 \\
\hline 6 & $13 / 01 / 06$ & $31 / 01 / 06$ & 18 & 6 & $10 / 01 / 06$ & $27 / 01 / 06$ & 17 \\
\hline 7 & $31 / 01 / 06$ & $22 / 02 / 06$ & 22 & 7 & $27 / 01 / 06$ & $21 / 02 / 06$ & 25 \\
\hline 8 & $22 / 02 / 06$ & $10 / 03 / 06$ & 16 & 8 & $21 / 02 / 06$ & $12 / 04 / 06$ & 50 \\
\hline 9 & $10 / 03 / 06$ & 05/04/06 & 26 & 9 & $12 / 04 / 06$ & $15 / 05 / 06$ & 33 \\
\hline 10 & 05/04/06 & 03/05/06 & 28 & 10 & $15 / 05 / 06$ & 08/06/06 & 24 \\
\hline 11 & 03/05/06 & $24 / 05 / 06$ & 21 & & & & \\
\hline 12 & $24 / 05 / 06$ & $21 / 06 / 06$ & 28 & & & & \\
\hline 13 & $21 / 06 / 06$ & $12 / 07 / 06$ & 21 & & & & \\
\hline
\end{tabular}


calcular a densidade de fluxo (q), utilizando a equação de Darcy-Buckingham, ou seja, a drenagem interna e a ascensão capilar diárias, $(q<0)$ e $(q>0)$, respectivamente:

$$
\vec{q}=-K(\theta) \cdot \overrightarrow{G r a d} \phi_{t}
$$

A variação da armazenagem de água no solo foi calculada pela diferença entre as armazenagens determinadas no início e no final de cada período. O método utilizado foi o gravimétrico (tradagem), cujas amostras de solo coletadas consistiram da camada de 0 a $0,15 \mathrm{~m}$ de profundidade, e dessa camada até $0,95 \mathrm{~m}$, de camadas contíguas de $0,10 \mathrm{~m}$ de espessura. Os conteúdos de água a base de massa foram transformadas a volume, utilizando a densidade do solo. As armazenagens foram calculadas pela regra trapezoidal:

$$
h_{Z}=\int_{0}^{0,90} \theta(Z) d Z \cong\left[1,5 \theta\left(Z_{1}\right)+\sum_{i=2}^{9} \theta\left(Z_{i}\right)-0,5 \theta\left(Z_{9}\right)\right] \times 0,10
$$

na qual $Z_{1}=0,075 \mathrm{~m} ; Z_{2}=0,20 \mathrm{~m} ; Z_{3}=0,30 \mathrm{~m}$; $Z_{4}=0,40 \mathrm{~m} ; Z_{5}=0,50 \mathrm{~m} ; \mathrm{Z}_{6}=0,60 \mathrm{~m} ; Z_{7}=0,70 \mathrm{~m}$; $Z_{8}=0,80 \mathrm{~m} \mathrm{e} Z_{9}=0,90 \mathrm{~m}$.

A $E T r$ foi calculada pela equação 3 , a partir dos demais componentes que foram medidos ou estimados.

\section{Análise dos dados}

A comparação dos dados de drenagem interna, ascensão capilar, evapotranspiração real e produtividade da cana-de-açúcar entre os tratamentos $\mathrm{T}_{1}$ e $\mathrm{T}_{2}$ do Município de Jaboticabal foi feita por meio do teste de Duncan (5\%), utilizando o programa estatístico SAS, Statistical Analysis System Institute (SAS, 2001).

\section{RESULTADOS E DISCUSSÃO}

Na figura 1 há uma representação dos valores médios da armazenagem de água no solo em função do tempo, para os tratamentos sem $\mathrm{N}\left(\mathrm{T}_{1}\right)$ e com $\mathrm{N}$ $\left(\mathrm{T}_{2}\right)$ do Município de Jaboticabal e para o tratamento com N $\left(\mathrm{T}_{2}\right)$ do Município de Pirassununga. Observase (Figura 1a) que a adubação nitrogenada praticamente não alterou o valor da armazenagem de água em todos os dias de amostragem. O período compreendido entre a segunda amostragem (18/10/ $2005)$ e a décima terceira (21/06/2006) corresponde aproximadamente ao estádio de formação da produção da cana-planta ou estádio de máxima utilização de água (Teramoto, 2003). As linhas paralelas na figura 1 são valores de armazenagem de água no perfil 0,0 $0,9 \mathrm{~m}$ de profundidade correspondentes a $-10 \mathrm{kPa} \mathrm{e}$ $-1.500 \mathrm{kPa}$ de potencial mátrico, também calculados pela equação (6), a partir dos dados do quadro 2. Considerando esse intervalo de potencial mátrico, praticamente durante todo o período de formação da produção, espera-se não ter faltado água para o crescimento e desenvolvimento das plantas em ambos os tratamentos. Percebe-se, também, pela figura 1a, que, da segunda à sétima amostragem, houve um período de recarga, e da sétima à décima terceira amostragem, um período de secagem do solo, o que significa que na primeira e última amostragens o solo armazenava praticamente a mesma quantidade de água.

$\mathrm{Na}$ área de Pirassununga (Figura 1b), o comportamento da armazenagem de água no solo foi semelhante ao da área de Jaboticabal em termos de disponibilidade de água, mas com distribuição diferente a partir do sexto período, em função da precipitação pluvial.

Com os valores diários das leituras dos tensiômetros nas profundidades $0,8 \mathrm{~m} ; 0,9 \mathrm{~m}$ e $1,0 \mathrm{~m}$, a função $\theta\left(\phi_{m}\right)$ na profundidade $0,9 \mathrm{~m}$ (equação 1) e a função $K(\theta)$ também na profundidade $0,9 \mathrm{~m}$ (equação 2 ), foram calculados os valores diários de drenagem interna $(D)$ ou ascensão capilar $(A C)$ na profundidade $0,9 \mathrm{~m}$ pela equação (5). A drenagem interna e a ascensão capilar diárias, juntamente com os valores diários de

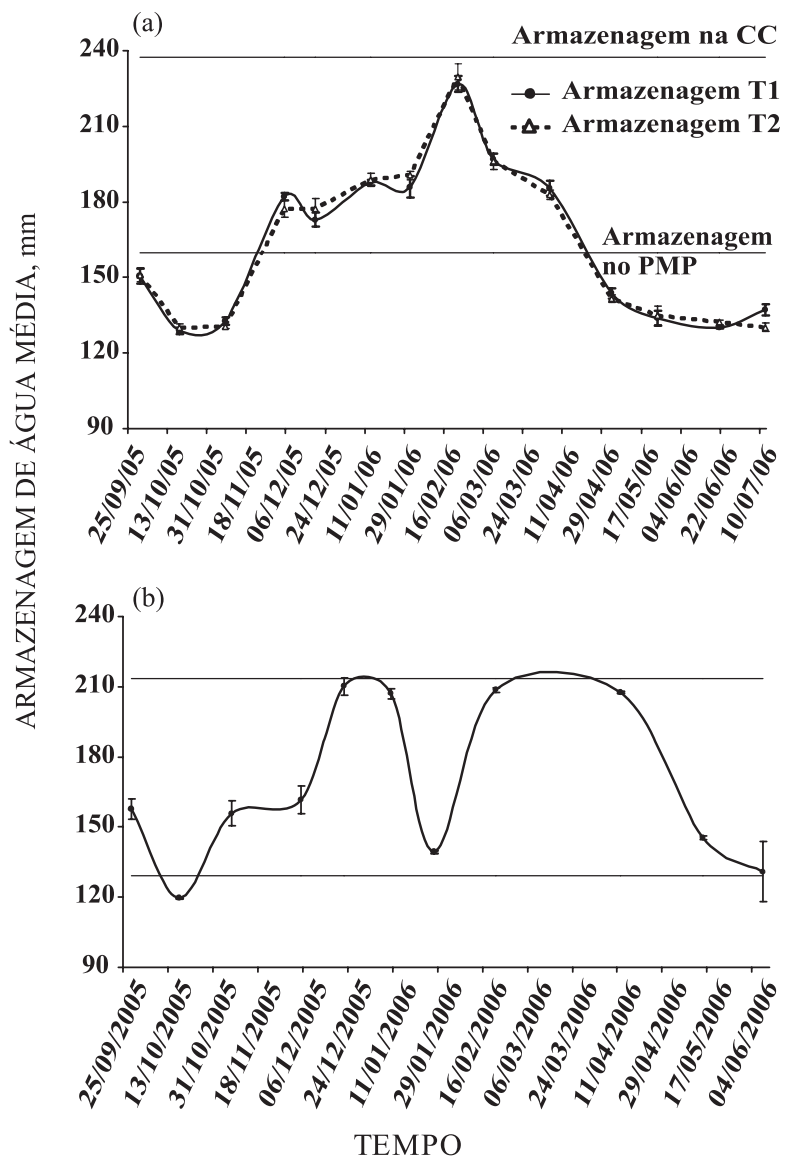

Figura 1. Valores médios da armazenagem de água no solo em função do tempo e seus respectivos erros-padrões, para os tratamentos sem nitrogênio $\left(T_{1}\right)$ e com nitrogênio $\left(T_{2}\right)$ do Município de Jaboticabal (a) e para o tratamento $\mathrm{T}_{2}$ do Município de Pirassununga (b). 
precipitação pluvial, acoplados às determinações da armazenagem de água no solo, a cada 20 dias aproximadamente, possibilitaram a estimativa da evapotranspiração real. Os valores obtidos para as variáveis da equação 3 encontram-se no quadro 4.

Observa-se que, em Jaboticabal, o somatório das drenagens internas e ascensões capilares resultou numa drenagem interna de $-186,4 \mathrm{~mm}$ no tratamento $\mathrm{T}_{1}$ e $-213,8 \mathrm{~mm}$ no tratamento $\mathrm{T}_{2}$, no final do período total estudado. Na área de Pirassununga, esse somatório foi de $-110,5 \mathrm{~mm}$. Analisando, separadamente, o somatório da quantidade de água drenada (D total) e o somatório da água que ascendeu (AC total) através do limite inferior à profundidade de $0,9 \mathrm{~m}$, houve, em Jaboticabal, uma AC total de 24,6 e 5,3 mm nos tratamentos $\mathrm{T}_{2}$ e $\mathrm{T}_{1}$, respectivamente; com relação à $\mathrm{D}$ total, foi de $-191,7 \mathrm{~mm}$, no tratamento $\mathrm{T}_{1}$, e $-238,4 \mathrm{~mm}$, no tratamento $\mathrm{T}_{2}$. Esses resultados sugerem que, na área do referido município, a adubação nitrogenada pode ter conduzido à maior desenvolvimento do sistema radicular, aumentando a extração de água e favorecendo maior ascensão capilar na época mais seca (períodos 12 e 13).

Nos períodos de maior precipitação pluvial, a drenagem interna foi maior no período 9, no qual ocorreu uma drenagem interna estatisticamente superior no tratamento $\mathrm{T}_{2}$ (teste de Duncan, a $5 \%$,
Quadro 4). Na área de Pirassununga, praticamente não houve ascensão capilar (AC total $=0,6 \mathrm{~mm}$ ) e D total $=-111,1 \mathrm{~mm}$.

Analisando o desvio-padrão dos resultados obtidos, nota-se, à semelhança de Cruz et al. (2005b), que trabalharam com laranja em solo parecido ao deste estudo, que a maior dispersão das estimativas do balanço de água ocorreu no processo drenagem interna e nos períodos de maior precipitação pluvial, sendo os erros desse processo propagados na estimativa da ETr. É importante observar também, entretanto, que o desvio-padrão da $E T r$ para o ciclo não atingiu $15 \%$ da ETr total. Os valores dos desvios-padrões das densidades de fluxo podem ter sido decorrentes da variação espacial das propriedades físico-hídricas do solo e da relação exponencial entre a condutividade hidráulica e o conteúdo de água no solo.

É difícil uma comparação entre as áreas porque, além dos solos não serem iguais (Quadros 1 e 2), a distribuição da precipitação pluvial também foi diferente entre elas, notadamente nos períodos 6 e 9 , nos quais praticamente não choveu em Pirassununga (Quadro 4). De qualquer maneira, é importante ressaltar que a drenagem interna é relativamente alta em ambas as áreas, alertando para problemas relacionados a uma potencial contaminação do lençol freático, em função do manejo da cultura.

Quadro 4. Precipitação pluvial (P), drenagem interna (D), ascensão capilar (AC) e evapotranspiração real $\left(E T_{r}\right)$ da cana-de-açúcar nos tratamentos, $T_{1}$ - sem adubação nitrogenada $\mathrm{T}_{2}$ - com adubação nitrogenada, avaliados ao longo dos períodos

\begin{tabular}{|c|c|c|c|c|c|c|c|c|c|c|c|}
\hline \multirow{2}{*}{ Per } & \multicolumn{4}{|c|}{ Jaboticabal $\left(\mathrm{T}_{1}\right)$} & \multicolumn{3}{|c|}{ Jaboticabal $\left(\mathrm{T}_{2}\right)$} & \multicolumn{4}{|c|}{ Pirassununga $\left(\mathrm{T}_{2}\right)$} \\
\hline & $\mathbf{P}$ & D/AC & $\mathbf{E T}_{r}$ & $\overline{\mathrm{ETr}}$ & D/AC & $\mathbf{E T}_{r}$ & $\overline{\text { ETr }}$ & $\mathbf{P}$ & D/AC & $\mathbf{E T}_{r}$ & ETr \\
\hline & \multicolumn{3}{|c|}{$\mathrm{mm}$} & $\mathrm{mm} \mathrm{dia}{ }^{-1}$ & $-\mathrm{m}$ & - & $\mathrm{mm} \mathrm{dia}{ }^{-1}$ & & $-\mathrm{mm}$ & ${ }^{\mathrm{r}}$ & $\mathrm{mm} \operatorname{dia}^{-1}$ \\
\hline 1 & 28,60 & $0,4 \mathrm{a}(3,0)^{(1)}$ & $50,6 \mathrm{a}(6,6)$ & 2,67 & $-3,7 \mathrm{a}(1,4)$ & $45,9 \mathrm{a} \quad(5,8)$ & 2,42 & 6,80 & $-0,1(0,1)$ & $44,9(5,8)$ & 2,24 \\
\hline 2 & 76,00 & $2,2 \mathrm{a}(3,0)$ & $74,3 \mathrm{a}(4,6)$ & 3,54 & $-1,7 \mathrm{a}(3,1)$ & $73,8 \mathrm{a} \quad(2,7)$ & 3,52 & 78,20 & $-0,2(0,1)$ & $41,7(7,5)$ & 1,98 \\
\hline 3 & 110,40 & $-7,6 \mathrm{a}(3,9)$ & $53,3 \mathrm{a}(6,7)$ & 1,98 & $-6,8 \mathrm{a}(15,9)$ & $56,9 \mathrm{a}(18,7)$ & 2,10 & 82,60 & $0,4(1,0)$ & $77,2(16,1)$ & 2,75 \\
\hline 4 & 43,80 & $-31,8 \mathrm{a}(11,7)$ & $21,2 \mathrm{a}(14,0)$ & 1,52 & $-24,3 \mathrm{a}(7,4)$ & $19,5 \mathrm{a} \quad(9,0)$ & 1,39 & 116,2 & $-0,4(0,3)$ & $67,3(14,0)$ & 3,95 \\
\hline 5 & 144,40 & $-11,6 \mathrm{a}(1,8)$ & $117,7 \mathrm{a}(6,1)$ & 4,71 & $-12,8 \mathrm{a}(2,0)$ & $120,2 \mathrm{a} \quad(6,1)$ & 4,80 & 238,4 & $-32,3(13,1)$ & $215,0(13,3)$ & 11,31 \\
\hline 6 & 104,80 & $-4,2 \mathrm{a}(1,5)$ & $102,5 \mathrm{a}(7,1)$ & 5,70 & $-4,4 \mathrm{a}(1,6)$ & $98,6 \mathrm{a} \quad(8,3)$ & 5,47 & 2,40 & $-0,5(0,4)$ & $69,5(3,6)$ & 4,08 \\
\hline 7 & 434,40 & $-110,2 \mathrm{a}(24,8)$ & $283,3 \mathrm{a}(24,8)$ & 12,87 & $-126,7 \mathrm{a}(49,2)$ & $268,5 \mathrm{a}(38,4)$ & 12,20 & 329,0 & $-59,1(28,2)$ & $213,9(29,6)$ & 8,55 \\
\hline 8 & 83,40 & $-4,8 \mathrm{a}(1,5)$ & $108,4 \mathrm{a}(9,5)$ & 6,78 & $-2,4 \mathrm{a}(3,2)$ & $114,6 \mathrm{a} \quad(4,2)$ & 7,15 & 262,0 & $-17,8(12,7)$ & $245,2(13,9)$ & 4,90 \\
\hline 9 & 176,20 & $-14,5 \mathrm{a}(4,1)$ & $173,0 \mathrm{a}(10,3)$ & 6,66 & $-44,6 \mathrm{~b}(21,2)$ & $144,8 \mathrm{~b}(24,7)$ & 5,57 & 0,0 & $-0,7(0,4)$ & $61,3(1,0)$ & 1,85 \\
\hline 10 & 6,80 & $-3,1 \mathrm{a}(11,8)$ & $45,3 \mathrm{a}(16,3)$ & 1,62 & $-11,0 \mathrm{a}(4,4)$ & $37,1 \mathrm{a}(7,9)$ & 1,33 & 3,0 & $0,2(0,2)$ & $31,2(29,0)$ & 1,95 \\
\hline 11 & 0,00 & $-1,4 \mathrm{a}(11,5)$ & $15,7 \mathrm{a}(5,5)$ & 0,75 & $6,7 \mathrm{a}(4,4)$ & $13,3 \mathrm{a} \quad(9,7)$ & 0,63 & & & & \\
\hline 12 & 0,00 & $2,8 \mathrm{a}(5,7)$ & $6,3 \mathrm{a}(3,2)$ & 0,23 & $16,6 \mathrm{a}(4,8)$ & $19,3 \mathrm{a} \quad(5,8)$ & 0,69 & & & & \\
\hline 13 & 12,80 & $-2,3 \mathrm{a}(2,2)$ & $5,6 \mathrm{a}(1,8)$ & 0,27 & $1,3 \mathrm{a}(2,1)$ & $16,0 \mathrm{a} \quad(4,6)$ & 0,76 & & & & \\
\hline Total & 1221,6 & $-186,4 \mathrm{a}(86,4)$ & $1057,8 \mathrm{a}(129)$ & - & $-213,8 \mathrm{a}(120,6)$ & $1028,6 \mathrm{a}(146,4)$ & 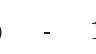 & 1118,6 & $-110,5(56,5)$ & $1067,1(134)$ & - \\
\hline \multicolumn{2}{|c|}{ AC total } & \multicolumn{2}{|l|}{$5,3(11,7)$} & \multicolumn{3}{|c|}{$24,6(11,3)$} & \multicolumn{5}{|c|}{$0,6(1,2)$} \\
\hline \multicolumn{2}{|c|}{ D total } & \multicolumn{2}{|l|}{$-191,7(74,7)$} & \multicolumn{3}{|c|}{$-238,4(109,2)$} & \multicolumn{4}{|c|}{$-111,1(55,3)$} & \\
\hline
\end{tabular}

(1) Os valores entre parênteses correspondem ao desvio-padrão; médias seguidas da mesma letra, na linha, não diferem entre si pelo teste de Duncan, a $5 \%$. 
É de real relevância a estimativa da evapotranspiração real (ETr) e sua relação com as condições físicas do solo e outros processos de movimentação da água no sistema solo-planta-atmosfera, além de processos fisiológicos ligados ao desenvolvimento radicular e foliar. A ETr seguiu a distribuição temporal do conteúdo de água no solo, decorrente principalmente da distribuição das chuvas.

$\mathrm{Na}$ área de Jaboticabal, a $E \operatorname{Tr}$ foi, em média, quase sempre menor ou aproximadamente igual no tratamento $\mathrm{T}_{2}$ em relação ao tratamento $\mathrm{T}_{1}$, embora menor estatisticamente apenas no período 9 (teste de Duncan, a 5 \%, Quadro 4), mas resultando numa ETr total média igual estatisticamente nos dois tratamentos $\left(1.057,8 \mathrm{~mm}\right.$ no $\mathrm{T}_{1}$ e $1.028,6 \mathrm{~mm}$ no tratamento $\mathrm{T}_{2}$ ), como indicado pela não-significância do teste $\mathrm{F}$ das produtividades médias de colmos industrializáveis nos dois tratamentos: no tratamento $\mathrm{T}_{1}$, esta produtividade foi de $144,827 \mathrm{t} \mathrm{ha}^{-1}$ (37.238 $\mathrm{kg} \mathrm{ha}^{-1}$ de massa seca de colmos) e, no tratamento $\mathrm{T}_{2}$, de 146,112 $\mathrm{t} \mathrm{ha}^{-1}\left(38.028 \mathrm{~kg} \mathrm{ha}^{-1} \mathrm{de}\right.$ massa seca de colmos).

Esses dados sugerem que a adubação nitrogenada não se traduziu num uso diferenciado de água pela cana-de-açúcar no ciclo cana-planta, visto que a eficiência do uso da água ( $E U A$, razão entre a produtividade e a ETr da cultura) no tratamento $\mathrm{T}_{1}$ foi de $13,7 \mathrm{~kg} \mathrm{~m}^{-3}$; no tratamento $\mathrm{T}_{2}$, de $14,2 \mathrm{~kg} \mathrm{~m}^{-3}$, em Jaboticabal, e em Pirassununga, a ETr total média foi de $1.067,1 \mathrm{~mm}$, com uma produtividade média de $141,25 \mathrm{t} \mathrm{ha}^{-1}$, apresentando, consequentemente, uma EUA de $13,26 \mathrm{~kg} \mathrm{~m}^{-3}$. Inman-Bamber \& Smith (2005) relataram pesquisas desenvolvidas com o objetivo de estudar a $E U A$ da cana-de-açúcar em diferentes regiões do mundo, utilizando métodos variados e medidas climáticas e fisiológicas, mostrando que a $E U A$ variou de 8,37 a 20,94 na Austrália (Kingston, 1994) e 4,8 a $12,1 \mathrm{~kg} \mathrm{~m}^{-3}$ na África do Sul, Hawaii e Austrália (Robertson \& Muchow, 1994). Observa-se que, embora o experimento de Pirassununga tenha sido instalado 44 dias antes do de Jaboticabal, e com o período total do estudo do balanço de água de 32 dias a menos, os valores de ETr nos dois locais foram semelhantes (Quadro 4).

Nesse contexto, Wiedenfeld (2000) verificou que os efeitos na produção estavam associados a períodos de maior demanda evapotranspirativa e de maior taxa de crescimento da cana-de-açúcar, ao passo que períodos de seis semanas de estresse hídrico não causaram reduções na produção, e o efeito do $\mathrm{N}$ foi reduzido ou eliminado em maiores períodos de estresse hídrico.

\section{CONCLUSÕES}

1. Nos períodos de alta pluviosidade, a drenagem interna foi significativa, alertando para possíveis problemas no manejo em relação a uma potencial contaminação do lençol freático.

2. Tanto a evapotranspiração real quanto a eficiência do uso da água foram da mesma ordem de grandeza, independentemente da adubação nitrogenada, e a armazenagem de água no solo foi praticamente a mesma no início e no fim do ciclo estudado.

\section{LITERATURA CITADA}

FNP Consultoria e Comércio. AGRIANUAL 2006: anuário da agricultura brasileira. Cana-de-açúcar. São Paulo, 2006. $504 \mathrm{p}$.

ANTONINO, A.C.D.; SAMPAIO, E.V.S.B.; DALL'OLIO, A. \& SALCEDO, I.H. Balanço hídrico em solo com cultivos de subsistência no semi-árido do Nordeste do Brasil. R. Bras. Eng. Agríc. Amb., 4:29-34, 2000.

ARAÚJO, W.F.; SAMPAIO, R.A. \& MEDEIROS, R.D. Irrigação e adubação nitrogenada em milho. Sci. Agríc., 56:909914, 1999.

BRUNINI, O.; GROHMANN, F. \& SANTOS, J.M. Balanço hídrico em condições de campo para dois cultivares de arroz sob duas densidades de plantio. R. Bras. Ci. Solo, 5:1-6, 1981.

CENTURION, J.F. \& ANDRIOLI, I. Regime hídrico de alguns solos de Jaboticabal. R. Bras. Ci. Solo, 24:701-709, 2000.

CINTRA, F.L.D.; LIBARDI, P.L. \& SAAD, A.M. Balanço hídrico no solo para porta-enxertos de citros em ecossistema de Tabuleiro Costeiro. R. Bras. Eng. Agríc. Amb., 4:23-28, 2000.

CRUZ, A.C.R.; LIBARDI, P.L.; CARVALHO, L.A. \& ROCHA, G.C. Balanço de água no volume de solo explorado pelo sistema radicular de uma planta de citros. R. Bras. Ci. Solo, 29:1-10, 2005a.

CRUZ, A.C.R.; LIBARDI, P.L.; CARVALHO, L.A. \& ROCHA, G.C. Evapotranspiração real de uma cultura de laranja em produção num Latossolo Vermelho-Amarelo. R. Bras. Ci. Solo, 29:659-668, 2005b.

EMPRESA BRASILEIRA DE PESQUISA AGROPECÁRIA EMBRAPA. Centro Nacional de Pesquisas de Solos. Sistema brasileiro de classificação de solos. Brasília, Embrapa Produção de Informação; Rio de Janeiro, Embrapa Solos, 1999. 412p.

FLINT, A.L. \& FLINT, L.E. Particle-size analysis. In: DANE, J.H. \& TOPP, G.C., eds. Methods of soil analysis: Physical methods. Madison, American Society of Agronomy, 2002. p.235-240.

GEE, G.W. \& OR, D. Particle size analysis. In: DANE, J.H. \& TOPP, G.C., eds. Methods of soil analysis: Physical methods. Madison, American Society of Agronomy, 2002. p.255-289. 
HILLEL, D.A.; KRENTOS, V.D. \& STILIANOV, Y. Procedure and test of an internal drainage method for measuring soil hydraulic characteristics in situ. Soil Sci., 114:395400, 1972.

INMAN-BAMBER, N.G. \& SMITH. D.M. Water relations in sugarcane and response to water deficits. Field Crops Res., 92:185-202, 2005.

KINGSTON, G. Benchmarking yield of sugarcane from estimates of water use. Proc. Austr. Sugar Cane Technol., 16:201-209, 1994.

LIBARDI, P.L.; REICHARDT, K.; NIELSEN, D.R. \& BIGGAR, J.W. Simple field methods for estimating soil hydraulic conductivity. Soil Sci. Soc. Am. J., 44:3-7, 1980.

LIBARDI, P.L. \& SAAD, A.M. Balanço hídrico em cultura de feijão irrigada por pivô central em Latossolo Roxo. R. Bras. Ci. Solo, 18:529-532, 1994.

MOURA, M.V.P.S.; FARIAS, C.H.A.; AZEVEDO, C.A.V.; DANTAS NETO, J.; AZEVEDO, H.M. \& PORDEUS, R.V. Doses de adubação nitrogenada e potássica em cobertura na cultura da cana-de-açúcar, primeira soca, com e sem irrigação. Ci. Agrotec., 29:753-760, 2005.

ROBERTSON, M.J. \& MUCHOW, R.C. Future research challenges for efficient crop water use in sugarcane production. Proc. Austr. Sugar Cane Technol., 16:193200, 1994.

SANTOS, A.C.; ANDRADE, A.P.; LIMA, J.R.S.; SILVA, I.F. \& CAVALCANTE, V.R. Variabilidade temporal da precipitação pluvial: Nível de nitrogênio no solo e produtividade de cultivares de girassol. Ci. Rural, 32:757764, 2002.

SAS Institute. SAS/STAT. User's guide, versão 8.2, Cary, 2001. 943p.

SIGRH: Banco de dados pluviométricos do Estado de São Paulo (Prefixo C4-070). Disponível em: < http:/ www.sigrh.sp.gov.br/ cgi-bin/bdhm.exe/plu>. Acesso em: 25 ago. 2008.
SOIL SURVEY STAFF. Keys to soil taxonomy. 9.ed. Washington, United States Department of Agriculture (USDA) - Natural Resources Conservation Service, 2003. $332 \mathrm{p}$.

STEWART, L.K.; CHARLESWORTH, P.B.; BRISTOW, K.L. \& THORBURN, P.J. Estimating deep drainage and nitrate leaching from the root zone under sugarcane using APSIM-SWIM. Agric. Water Manag., 81:315-334, 2006.

TERAMOTO, E.R. Avaliação e aplicação de modelos de estimativa de produção de cana-de-açúcar (Saccharum spp) baseados em parâmetros do solo e do clima. Piracicaba, Universidade de São Paulo, 2003. 85p. (Tese de Doutorado)

TIMM, L.C.; OLIVEIRA, J.C.M.; TOMINAGA, T.T.; CÁSSARO, F.A.M.; REICHARDT, K. \& BACCHI, O.O.S. Water balance of a sugarcane crop: Quantitative and qualitative aspects of its measurement. R. Bras. Eng. Agric. Amb., 6:57-62, 2002

TRIVELIN, P.C.O.; VITTI, A.C.; OLIVEIRA, M.W.; GAVA, G.J.C. \& SARRIÉS, G.A. Utilização de nitrogênio e produtividade da cana-de-açúcar (cana-planta) em solo arenoso com incorporação de resíduos da cultura. R. Bras. Ci. Solo, 26:637-646, 2002.

van GENUCHTEN, M.T. A closed-form equation for predicting the hydraulic conductivity of unsaturated soils. Soil Sci. Soc. Am. J., 44:892-898, 1980.

VITTI, A.C.; TRIVELIN, P.C.O.; GAVA, G.J.C.; PENATTI, C.P.; BOLOGNA, I.R.; FARONI, C.E. \& FRANCO, H.C.J. Produtividade da cana-de-açúcar relacionada ao nitrogênio residual da adubação e do sistema radicular. Pesq. Agropec. Bras., 42:249-256, 2007.

WATSON, K.K. An instantaneous profile method for determining the hydraulic conductivity of unsaturated porous materials. Water Res. Res., 2:709-715, 1966.

WIEDENFELD, R.P. Water stress during different sugarcane growth periods on yield and response to $\mathrm{N}$ fertilization. Agric. Water Manag., 43:173-182, 2000. 
